\title{
A hierarchical phasing algorithm for multi-element optical interferometers
}

\author{
E. Pedretti ${ }^{1, \star}$ and A. Labeyrie ${ }^{2}$ \\ ${ }^{1}$ Collège de France \& University of Manchester, Nuffield Radio Astronomy Laboratories, Jodrell Bank, UK-Cheshire, \\ SK11 9DL, UK \\ ${ }^{2}$ Collège de France et Observatoire de Haute-Provence, CNRS, F-04870 Saint-Michel l'Observatoire, France
}

Received February 26; accepted March 26, 1999

\begin{abstract}
Large multi-aperture interferometers are expected to provide snap-shot images with milli- to nanoarcsecond resolution, directly at the combined focus. This requires a densified exit pupil and phased beams for all the wavelengths used. The wave analysis methods serving for adaptive optics in monolithic telescopes being inadequate for diluted apertures, we have developed a different phasing algorithm. It involves a hierarchy of aperture triplets and uses image sharpness criteria. Numerical simulations on unresolved and resolved objects show that a single iteration is generally sufficient. On wavelength-dependant objects, the proper position indexing of the monochromatic image components appears also efficiently achievable.
\end{abstract}

Key words: instrumentation: interferometers - methods: numerical - techniques: image processing - techniques: interferometric - telescopes

\section{Introduction}

Long baseline optical interferometry is still in its infancy, with aperture count currently limited to 3 . The feasibility of using numerous apertures, spaced kilometers apart, to obtain high-resolution snapshot images was initially debated (Traub 1986; Beckers 1986, 1997). Fizeau interferometry, the only beam-combining scheme then known to provide snap-shot images did not appear usable with highly-diluted apertures. Only recently was a solution found (Labeyrie 1996), in the form of "densified-pupil imaging", a beam-combining scheme which can achieve efficient imaging on compact objects.

With this scheme, ways of using diluted apertures spanning up to $100000 \mathrm{~km}$ are foreseen (Labeyrie 1998). It will require active or adaptive phasing, like in monolithic telescopes.

\footnotetext{
* Now at Observatoire de Haute-Provence - CNRS.
}

The closure phase method (Jennison 1958), widely used in the radio interferometry domain and also recently used in the COAST optical interferometer (Baldwin et al. 1996) for observing the binary star Capella, is suitable for phasing diluted arrays but requires individual phase measurements on all the baselines taken into account for the closures. In our case, this would imply a pair wise recombining technique for a number of baselines much larger than the 13 groups of apertures used for our approach to cophasing the array.

We have developed a different algorithm, suggested by one of us (A.L.), and possibly well suited for this type of instrument. We describe the algorithm in Sect. 4, results of numerical simulations in Sect. 5, and discuss the limitations in Sect. 6.

\section{Simulated images from a diluted array}

We considered the case of densified-pupil interferometers (Labeyrie 1996), a new class of instruments where beams are combined like in Michelson's 20- and 50-feet systems, but with more than 2 beams. The densified-pupil concept evades the "golden rule", once thought inescapable, which stated that imaging interferometers had to be Fizeau-like (Labeyrie 1983; Traub 1986; Beckers 1986). Fizeau-type interferometers having many apertures can produce snapshot images, but not if the aperture is highly diluted since most energy then goes in the numerous side-lobes rather than in the central interference peak. Densifying the exit pupil (Fig. 1), by extending to many apertures the kind of "periscopic" arrangement originally used by Michelson, can concentrate the energy in the interference peak, but appeared to destroy the isoplanaticity needed to observe resolved objects.

The solution recently found uses a restricted class of Michelson-type arrangements where:

- the pattern of sub-aperture centres is identical in the entrance and the exit pupils; 


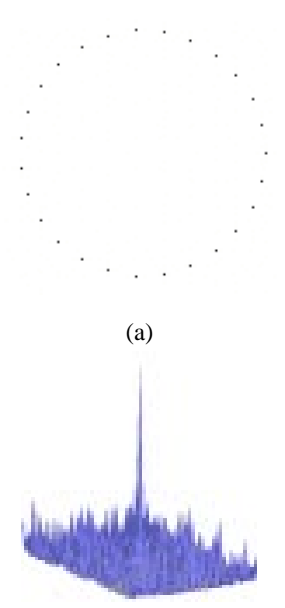

(c)

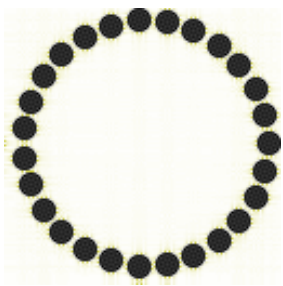

(b)

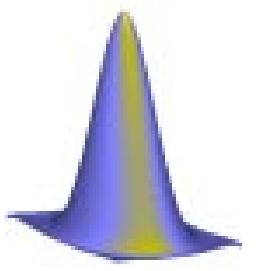

(d)

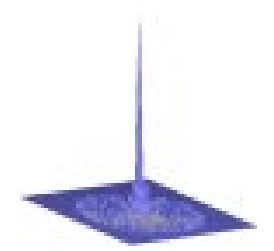

(e)
Fig. 1. Image formation with a densified-pupil array. The array's exit pupil is the convolution of an array of Dirac peaks a), with a sub-pupil. The Fourier transform of the peak array c), called the interference function, here has a central peak surrounded by 54 lower peaks. The broad multiplicative envelope, called the diffraction function, shown in d) is the Fourier transform of the sub-pupil, made proportionally larger in the exit pupil than in the entrance aperture. The image of a nonresolved star is obtained in e) as the product of the patterns c) and d). With a resolved star, only the interference function is becoming convolved with the star function if the pupil is highly densified

- all sub-pupils are of homogeneous size and shape.

It can provide full energy concentration in the combined high-resolution image, but the field and object size are restricted to about $N \times N$ resolved elements, if $N$ is the number of apertures.

If the exit pupil is highly densified, as is necessarily the case in large space instruments such as the proposed ExoEarth Discoverer (Boccaletti et al. 1999), the Terrestrial Planet Finder (Angel \& Woolf 1997; Woolf 1997) and the Exo-Earth Imager (Labeyrie 1998), then a valid approximation for the monochromatic intensity distribution $B(x, y)$, in the combined focal image was shown to be (Labeyrie 1996):

$B_{(x, y)} \approx \gamma_{d}^{-2} A_{(x, y)}\left[O_{\left(\frac{x}{\gamma_{d}}, \frac{y}{\gamma_{d}}\right)} \otimes I_{(x, y)}\right]$,

where $O_{(x, y)}$ is the intensity distribution of the object. $I_{(x, y)}$ is the interference function of the interferometer, i.e. the Fourier transform of an array of Dirac peaks located at the sub-aperture centres, $A_{(x, y)}$ the diffraction pattern from one sub-aperture and $\otimes$ is the convolution symbol. Finally, $x$ and $y$ are the coordinates in the focal plane. The quantity $\gamma_{d}=\left(d_{\mathrm{o}} / D_{\mathrm{o}}\right) /\left(d_{i} / D_{i}\right)$ describes the amount of pupil densification, $D_{i}$ and $d_{i}$, being the pupil and sub-pupil sizes at the entrance, while $D_{\mathrm{o}}$ and $d_{\mathrm{o}}$ are the corresponding quantities at the exit pupil.

Equation (1) shows that the interference function $I_{(x, y)}$ (Fig. 1c) is convolved with the object's intensity distribution and then multiplied by the wide diffraction pattern of the sub-pupil (Fig. 1d). For the large values of the pupil densification this latter pattern is considered insensitive to object position and simply behaves, where Eq. (1) is valid, as a windowing function which multiplies the convolution.

$I_{(x, y)}$ generally has a central peak if the sub apertures are phased, a desirable situation which improves greatly the quality of the snapshot image $B_{(x, y)}$ (without phasing, speckle interferometry can still produce images). In monochromatic light, the phasing is required modulo $2 \pi$ radians, but reaching the phasing condition at numerous wavelengths simultaneously requires all optical paths to be equal, as is the case for a perfect giant telescope. In our simulation we used 3 wavelengths to reduce the path differences from $10 \mu \mathrm{m}$ to nearly zero. The example discussed here involves 27 identical apertures, arrayed along a circle, but the algorithm can be extended to any hierarchy of triplets arbitrarily arrayed.

\section{Phasing a hierarchy of triplet apertures}

An early algorithm for adaptive optics, not relying on wavefront continuity, was proposed by Muller \& Buffington (Muller \& Buffington 1974). It is based on the maximisation of a sharpness function, defined as:

$S=\int B_{(x, y)}^{2} \mathrm{~d} x \mathrm{~d} y$

where $B$ is the intensity of the image at a point $(x, y)$.

The mirror actuators are adjusted each in turn, with repeated iterations performed faster than the seeing lifetime, to maximise the sharpness function. The convergence however tends to be trapped in secondary maxima. To overcome this problem, Ribak (Ribak 1990) used simulated annealing: noise is added to the actuator signals and gradually reduced until the system falls in a state of minimum energy, reached when the sharpness function is maximised.

Hamaker et al. (1977) have later remarked that, according to Parseval's theorem, maximising the sharpness function is equivalent to maximising the integrated square modulus in the image's Fourier transform, which is the pupil's auto-correlation function multiplied by the object's Fourier transform. The maximum occurs for uniform pupil phases. However, if the aperture is diluted, the intensity of the autocorrelation peaks remains invariant if they do not overlap. This indicates that the sharpness approach cannot work if the exit pupil aperture is highly diluted, as would be the case for long-baseline Fizeau interferometers. The densified exit pupils considered here can be made to meet the peak overlap requirement.

We have tried to improve the convergence and to avoid the simulated annealing, by using a hierarchy of aperture triplets. The 27-aperture interferometer is initially 


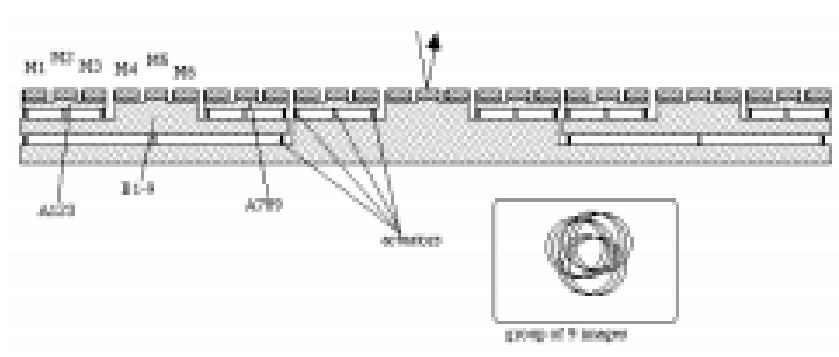

Fig. 2. Hierarchical arrangement of actuators carrying 27 mirrors for phasing according to the triplet algorithm. For clarity, the system is sketched as a line array, and without the tiptilt correctors carrying each elementary mirror. Each triplet of mirrors is carried by a plate, itself carried by 3 actuators, arranged as a triangle to provide piston and tip-tilt corrections. 3 such plates, carrying a total of 9 mirrors, are themselves carried by a larger plate, also carried by 3 actuators. A group of 9 mirrors can be phased by adjusting both lateral triangle plates, in terms of tip-tilt and of piston. The criterion used for this 6-parameter adjustment is the sharpness value. If the star is unresolved, centering the honeycomb fringe patterns from each mirror triplet, and the finer honeycomb from the triplet of triplets is also suitable. Once the 3 groups of 9 mirrors are independently phased, the global phasing of 27 mirrors can be obtained similarly by applying tip-tilt and piston corrections to both lateral plates carrying 9 mirrors. Finally, the diffraction functions from each elementary mirror may be centered with their tip-tilt actuators (not shown). Phasing is achieved modulo $2 \pi$, but the use of several wavelengths can remove the ambiguity and provide zero path difference

divided in 9 separate interferometers, each combining images from 3 adjacent apertures, then re-arranged as 3 separate interferometers having 9 apertures each and finally re-arranged as a single interferometer having 27 apertures. If observations are performed from Earth the steps of rearrangements are cycled at high speed, within the lifetime of seeing, by tilting elements of the active mirror support to "explode" the image into separated triplet components. More delicate tip-tilt and piston adjustments of the same elements provide the phase adjustments, as described in Fig. 2.

The monochromatic interference pattern from $3 \mathrm{sub}-$ apertures is generally a honeycomb-like interference function, multiplied by the diffraction function. Adding 3 such patterns from adjacent groups creates a finer honeycomb pattern within the coarse one. The triplet grouping can be pursued at higher levels of the hierarchy to include 27, 81, 243 , etc. apertures. The closest apertures are grouped first to benefit from the fact that the object is less resolved.

For a non-resolved star, phasing is directly achievable by centering a cell of the honeycomb pattern appearing at each level of triplet formation. This requires adjusting 2 phases, those of 2 among the 3 groups of apertures at every step of the routine (Fig. 3). In monochromatic light and for 3 groups of apertures, the honeycomb pattern can be centered by extracting the phase values in the 6 peaks of its

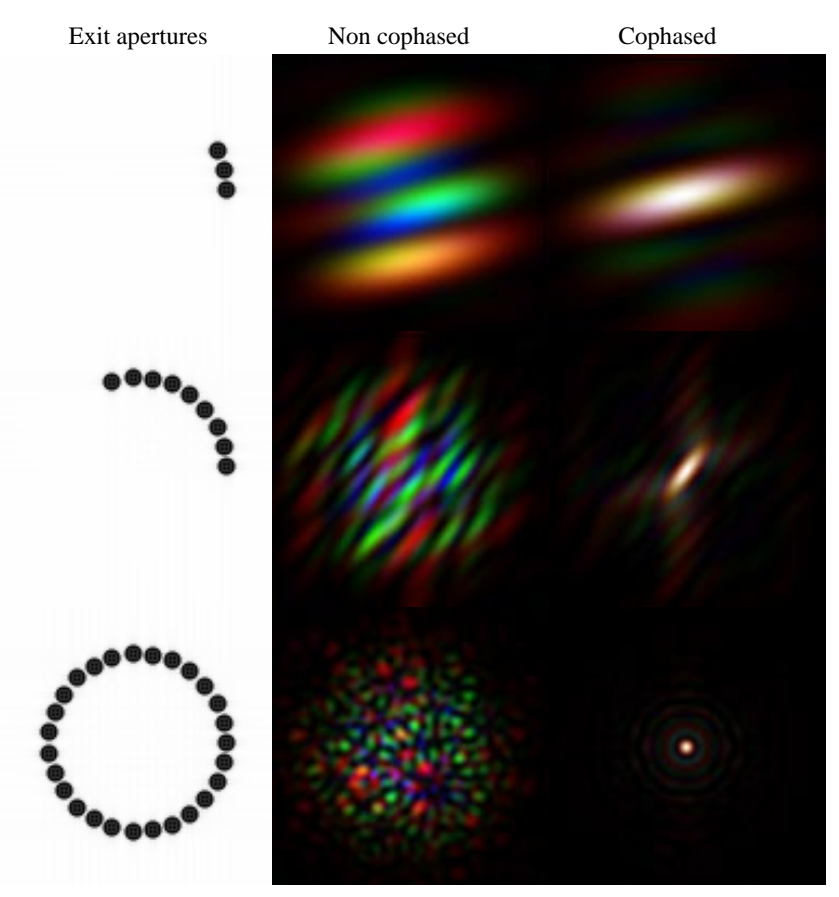

Fig. 3. Steps of phasing sequence for 27 apertures, using 3 wavelengths on a point source. Top row: 3 elements of the entrance aperture (top-left) and their interference pattern obtained before (middle) and after (right) correcting optical path errors. Middle row: combining 3 such groups of apertures provides finer fringes, which can be centered by adjusting the global phases of 2 groups. Bottom row: one more similar step corrects the optical path errors among the 27 apertures, thus producing a narrow interference peak. With a continuous spectrum rather than just 3 wavelengths, the speckles of the central bottom image would be appreciably smoothed

Fourier transform. In polychromatic light, 3-dimensional Fourier transforms of the $x, y, \lambda$ data cubes provided by the spectro-imaging camera can provide similar information with full removal of the modulo $2 \pi$ phase ambiguity. This algorithm has not been tested yet, but a 2-aperture analog is routinely used at the "Grand Interféromètre à 2 Télescopes" (GI2T) (Morel \& Koechlin 1998) for fringe acquisition.

More simply, the central white cell appearing in a honeycomb pattern amidst the coloured lateral cells can also be identified, even with a broad-band monochrome camera, from its higher contrast. Centering all the white cells from the various triplet groups onto a common fiducial mark corrects directly the optical path errors. On resolved objects, such honeycomb centering should not generally succeed since de-centered honeycomb components can be present. Indeed, imaging an object through a diluted triplet aperture causes a convolution with the honeycomb-like spread function. If the object is itself a honeycomb-like grid pattern matching this function, then the convolution preserves the image contrast, a situation which may be qualified as "honeycomb resonance". With 3 different triplets now forming a single aperture, 
3 different grid components of the object add their images, but they do not generally have a common centering. Hence the need for another criterion, sharpness for example, to adjust the tip-tilt of 2 pupil triplets before adjusting their global piston. Six parameters should therefore be adjusted at each level in the hierarchical procedure.

When calculating the sharpness integral, the diffraction envelope of the sub-images causes unwanted biases, which tend to center the brighter zones. The bias can be avoided by "flattening" the diffraction peak: each recorded sub-image must be first divided by the known diffraction function, within the peak area.

Somewhat surprisingly, our simulations (Fig. 4) on resolved objects worked well without these precautions, using a simplified version of the routine: only piston adjustments were made at each level, and no flattening of the diffraction envelope was carried out. It is however likely that some object shapes require the complete routine. Also, no trapping in secondary maxima of the sharpness function was observed, but the reasons are unclear. Further investigations are desirable in these respects.

A single camera can receive all partial images and the fully combined image, using either temporal or spatial separations as shown in Fig. 5. Rather than using the actuators for exploding the image into the various groups at a fast cycling rate, beam-splitters can provide a parallel display of these triplet, ninetuplet, etc. groups (Labeyrie 1998). Mapping the $x, y, \lambda$ information is efficiently achievable with a spectro-imaging attachment such as the combination of a grism and a micro-lens array developed by Bacon et al. (1995) at the Observatoire de Marseille.

\section{Phasing and path difference equalisation}

The sharpness $S$ was maximised by exploiting its sine response to phase variations (Muller \& Buffington 1974). In polychromatic light, the algorithm is applied separately to the different wavelength components in the $x, y, \lambda$ data cube.

The set of pupil phases is thus obtainable at each wavelength, and the modulo $2 \pi$ ambiguity can be removed to find the true optical path deviations and correct them. Only in such conditions of ideal interferometer adjustment can one obtain white images directly at the combined focus, with perfect colour registration and including any spectral components too faint for being separately imageable with the monochromatic algorithm. On resolved and colour-dependant objects, ensuring zero optical differences is made more difficult by the a priori unknown position indexing among the monochromatic images. One needs to solve for the value of integer $k$ in Eq. (3)

$\delta=(k+\epsilon) \lambda+(\alpha x+\beta y)$,

where $\epsilon=\phi / 2 \pi$ and $\lambda$ is the wavelength. The term $\alpha x+\beta y$, where $\alpha, \beta$ are the sub-pupil's position coordinates, is the path difference generated by the wavelength-dependant image position error $x, y$. For a non-resolved star the shift $x, y$ is identical at every wavelength and the second term of Eq. (3) therefore vanishes. One may then apply the classical fractional excess algorithm, used in the field of interferometric metrology since Michelson's measurement of the standard metre, to calculate the optical path differences. It consists in calculating, at every wavelength, $\delta$ values corresponding to all possible values of the unknown integer $k$. A $\delta$ value re-appearing at all wavelengths is the correct value of the optical path error for the sub-pupil considered. We have used this type of method, rather than the 3-dimensional Fourier transform, and verified the expected performance. Although not expected to work on resolved, colour dependant, objects, it also succeeded with such objects in most cases (Figs. 4, 5).

It appears possible to extend the method for dealing also with the shift term in Eq. (3), as required for full adjustment on resolved, colour-dependant, sources. Because the $x, y$ image shift is identical for all sub-pupils, at a given wavelength, the corresponding equations are coupled and appear solvable if enough wavelengths are used. Different values of $x, y$ and again the integer $k$ can be inserted in the series of equations obtained at all wavelengths. The candidate values of $\delta$ thus obtained may be organised as a 2-dimensional array of cubes, each being derived from a pair of vectors $x_{\lambda}, y_{\lambda}$, specifying a trial set of position shifts. The cube axes are divided in units of $k$, $\lambda$ and the sub-pupil number. The particular cube providing the correct set of optical path errors is identified from the presence of numbers repeating in all lines within each of its $k, \lambda$ planes.

No attempt has yet been made to apply this method, although it may also solve classical problems of source position indexing in radio interferometry.

\section{Results}

Figure 4 shows one of the objects used in the simulation, a coloured cluster of 27 stars. The object's intensity distribution was convolved with the interference function of the interferometer and then multiplied with the diffraction function, both functions being converted to intensities. The optical path differences were found, using 3 wavelengths, with initial wavefront errors of the order of $10 \mu \mathrm{m}$. The Strehl ratio, i.e. the peak intensity in the image of a point source, relative to the case of perfect phasing, gives an idea of the phasing performance. It reaches $30 \%$ with 2200 photons detected during the phasing cycle and $80 \%$ with $1810^{6}$ photons.

The successive frames of Fig. 6 from left to right depict changes in the full combined image during the phasing steps for the same object.

The field and object size restriction with densifiedpupil imaging often implies that the object is unresolved 


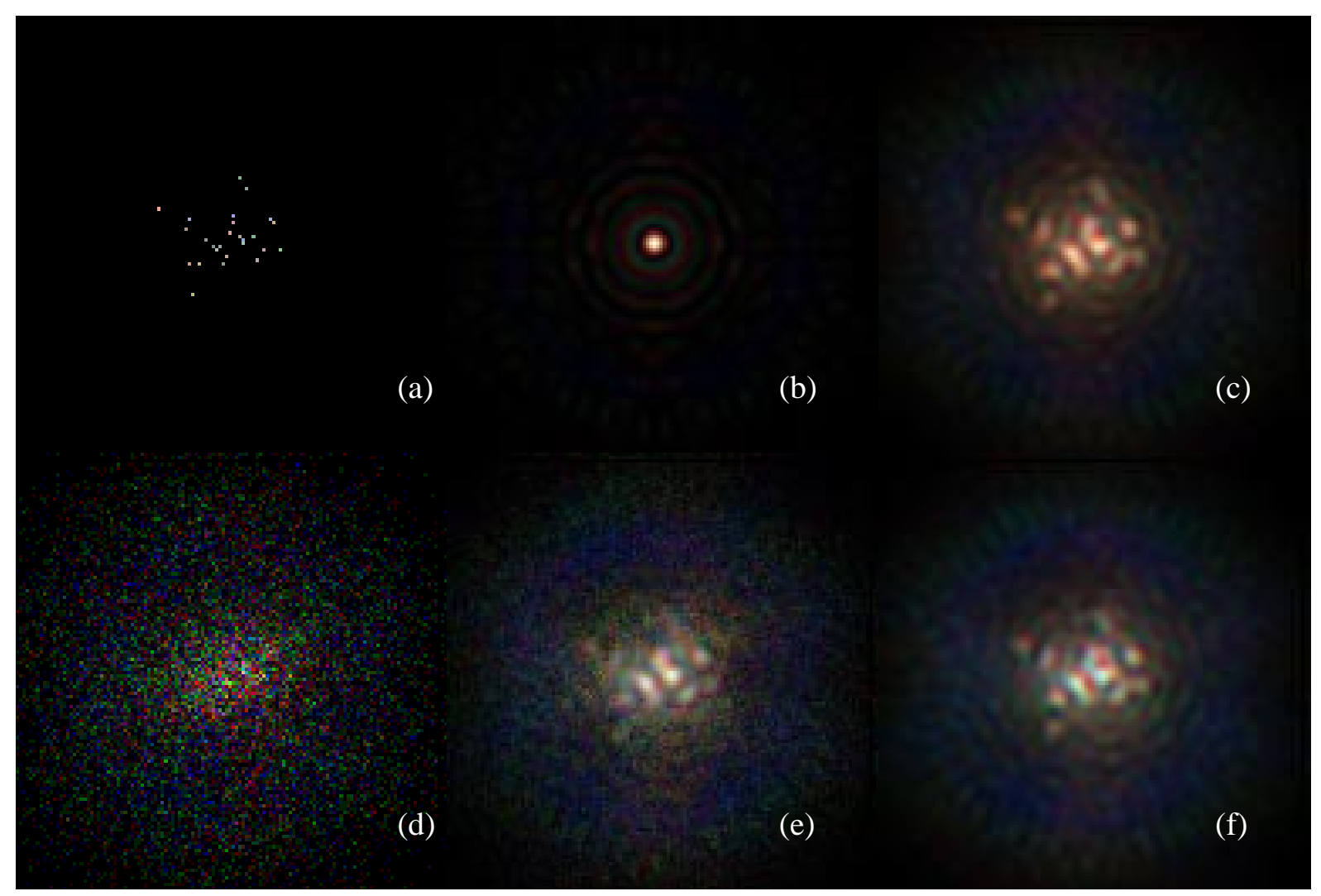

Fig. 4. Effect of photon noise on the phasing algorithm; a) simulated star cluster having 27 stars of different colour; b) interference function; c) theoretical image with zero path difference. The images were obtained with a single iteration of the phasing algorithm; d) image obtained with 2200 total photons obtained from 3 wavelengths, Strehl ratio $=0.3 ; \mathbf{e}$ ) case of 29000 photons, Strehl ratio $=0.7 ; \mathbf{f})$ case of $1810^{6}$ photons, Strehl ratio $=0.8$

or little resolved by the smallest baselines. Although the simplified routines which we used in the simulations did not include all the features which appeared necessary, the resulting imaging quality approached the theoretical limits on the source shapes used, typical of resolved stellar and galactic objects. Even better results should therefore be expected with future refined versions of the algorithm.

\section{Limiting magnitude}

The limiting magnitude estimates given by the early proponents of the sharpness criterion (Muller \& Buffington 1974; Dyson 1978) were lower than those obtained in monolithic adaptive telescopes. This can be attributed to the intrinsic advantage of exploiting the wavefront continuity with analyser systems such as Shack-Hartmann (Shack \& Platt 1971) or curvature sensing (Roddier \& Roddier 1991). Long-baseline interferometers obviously cannot benefit from this advantage, but the triplet algorithm appears to improve somewhat the limiting magnitude with respect to the original sharpness algorithm.

Photon noise in the recorded image generates a speckled halo in its Fourier transform. The halo's addition to the correlation peaks affects their phase and intensity.
With a photon-counting camera as the image detector, the Poisson statistics of the detected photons is preserved. If $p$ photons are detected during a short exposure, the image's Fourier transform has a central peak having $p$ times the average intensity of the halo. As in the high-level pattern, the intensity of the surrounding correlation peaks relative to the central peak is $N^{-2}$ and further attenuated if the star is resolved. To have correlation peaks emerging from the halo $p>N^{2}$ photons are thus needed, as required for reasonably accurate determination of the phases.

If the star is unresolved, the aperture phases can be calculated directly from the phases of the correlation peaks. Simulations with a simple iterative routine provided a fast convergence ( 2 or 3 iterations) and verified the $p>N^{2}$ condition. This was achieved by subtracting from the phase of each aperture the average phase from the $N$ correlation peaks related to the aperture, and iterating after recalculating the image's Fourier transform. In polychromatic light, the fractional excess method can be applied to the phases obtained in each correlation peak for deriving the optical path in each peak, and the routine then provides the optical paths of the apertures.

The 6-parameter optimisation of sharpness for each triplet requires 2 exposures for adjusting each 


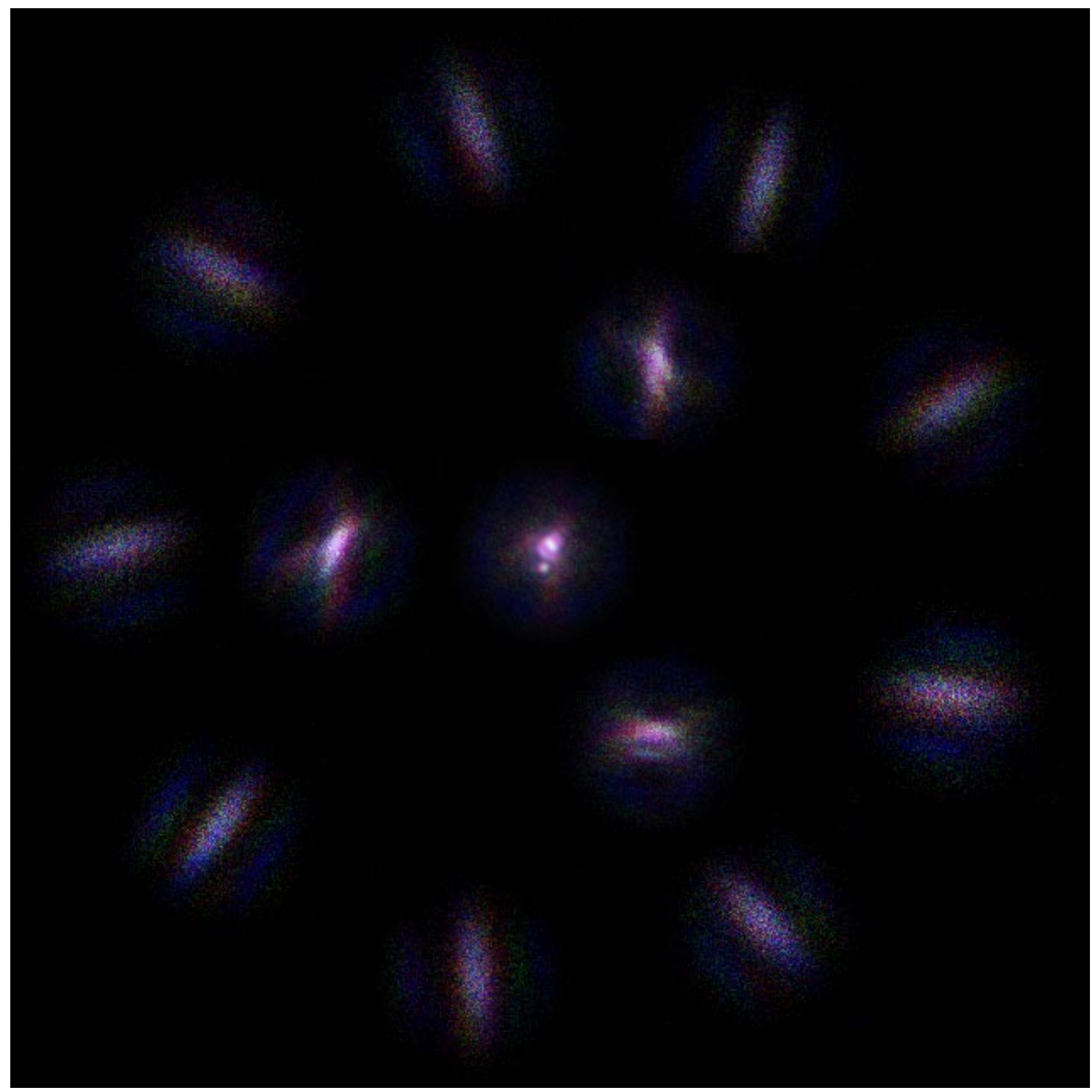

Fig. 5. Picture of partial and fully combined images with 9 groups of 3 apertures (external ring), 3 groups of 9 (intermediate ring), 1 group of 27 (centre), and the corresponding diffraction patterns, as they would appear when formatted on a single camera. The object used in the simulation is the galaxy M 51. The simulation was performed with photon noise. In the final image a Strehl ratio of 0.84 was obtained with 250000 photons

parameter and thus 12 exposures. This requires $12 \times 9^{2}=972$ detected photons from 9 apertures. Similarly, the full aperture stage requires $12 \times 27^{2}$ photons, hence a total of about $p=14000$ within the life-time of seeing. In practice fewer photons, about 800 , proved enough to obtain rather good images in our simulations of non-resolved objects, but resolved objects are less favorable. This number also matches the $p>N^{2}$ condition mentioned above for direct phase retrieval on un-resolved stars. On a resolved object such as shown in Fig. 7d, 130000 photons were needed to obtain a Strehl ratio of 0.96 with a single wavelength.
With $p$ photons received from the $N$-element aperture in time $\tau$, corresponding to the life-time of seeing, the incident photon flux per square centimeter $B$ is: $B=4 p /\left(\pi d^{2} \tau N \eta\right)$ and the stellar magnitude $m_{v}=2.5 \log (734 \delta \lambda / B), d$ being the size of the subaperture elements in centimeters, $\eta$ the overall quantum efficiency, and $\delta \lambda$ the bandwidth in Angström units. The limiting magnitude found with 27 apertures of $10 \mathrm{~cm}, 0.02 \mathrm{~s}$ seeing lifetime, $\eta=0.1$ and $300 \mathrm{~nm}$ optical bandwidth is 5.4 with $p=14000$ photons and 8.3 with $p=800$ photons, somewhat higher than the value 4.3 found by Muller et al. in identical conditions. 


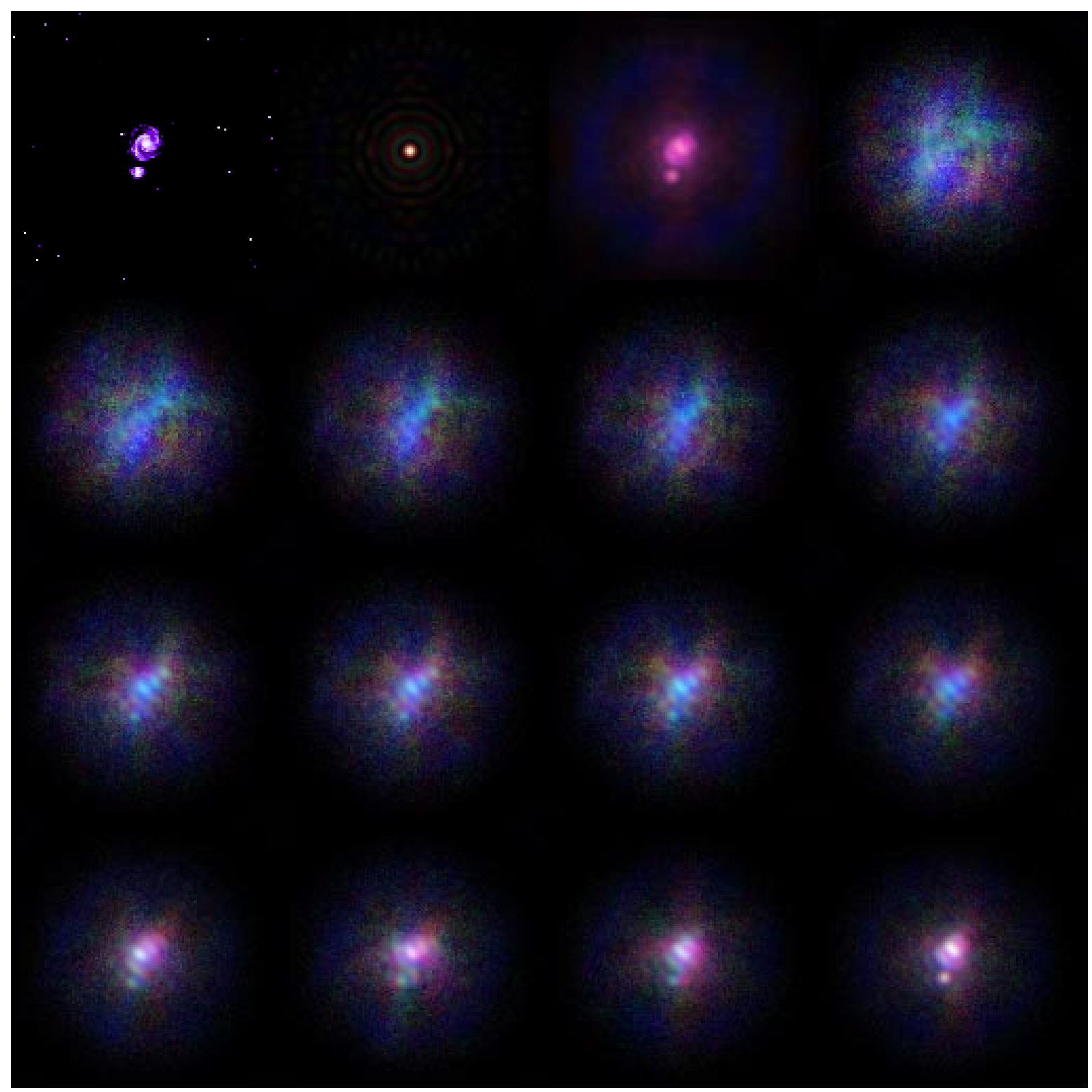

Fig. 6. Simulated phasing sequence on galaxy M 51, in presence of photon noise. The galaxy is shown in the top-left frame, followed by the interference function of the interferometer and the convolution of the object with the interference function. The object is unresolved by the smallest baselines, its components have different colours and phasing is achieved with 250000 photons. Successive frames depict changes in the full combined image during the phasing steps. The last frame, at bottom right, is the final step of the phasing process, where a Strehl ratio of 0.80 was achieved

Large sub-apertures with uniform phase, obtainable with a number of large ground-based telescopes equipped with adaptive optics and a laser star, can push the limiting magnitude for inter-aperture phasing towards $m_{v}=20$, assuming $10 \mathrm{~m}$ telescopes. The intra-aperture adaptive optics increases the lifetime of the wavefront's phase, owing to its averaging effect, and this contributes to the magnitude gain.

In space, much longer lifetimes can be expected, given the excellent predictability and measurability of telescope motions in the quiet micro-gravity environment. The availability of reference stars also favors efficient phasing on very faint objects. As discussed elsewhere (Labeyrie 1998), the extreme case of $100000 \mathrm{~km}$ optical baselines dedicated to observations of neutron stars appears manageable in terms of phasing. A more detailed analysis of limiting magnitudes with various array configurations, object shapes and phasing methods is desirable. 


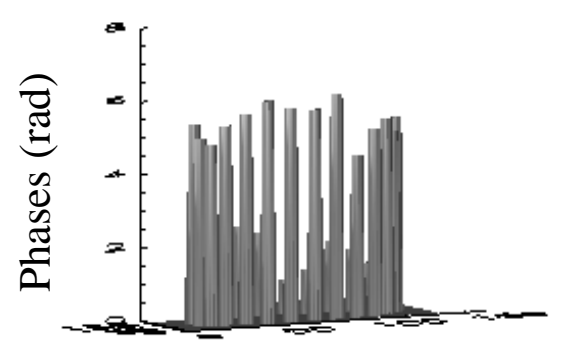

(a)

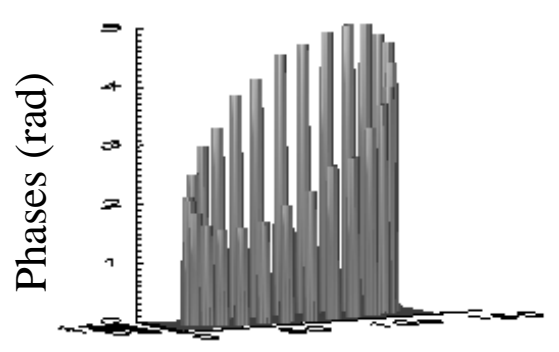

(b)
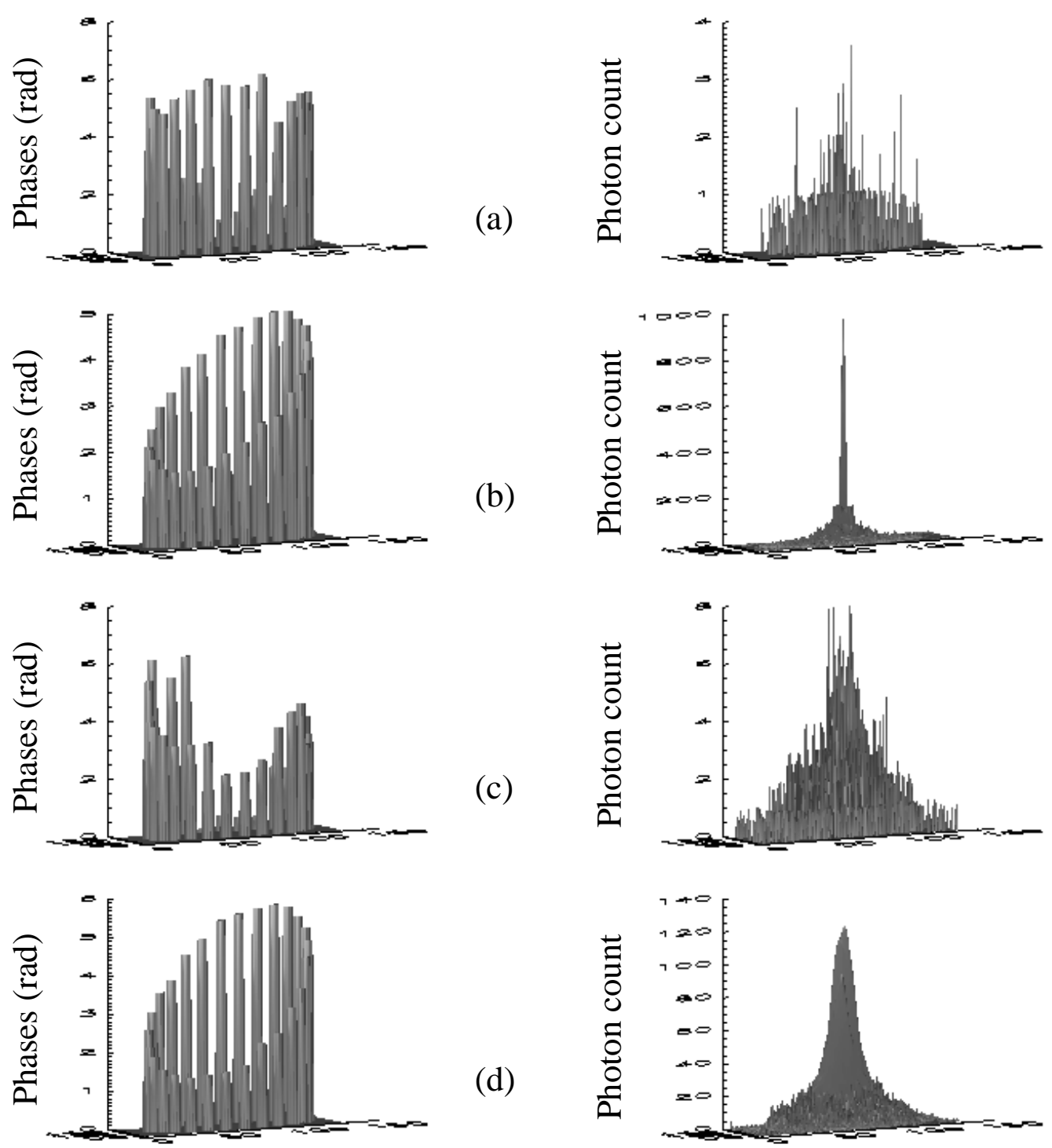

(c)
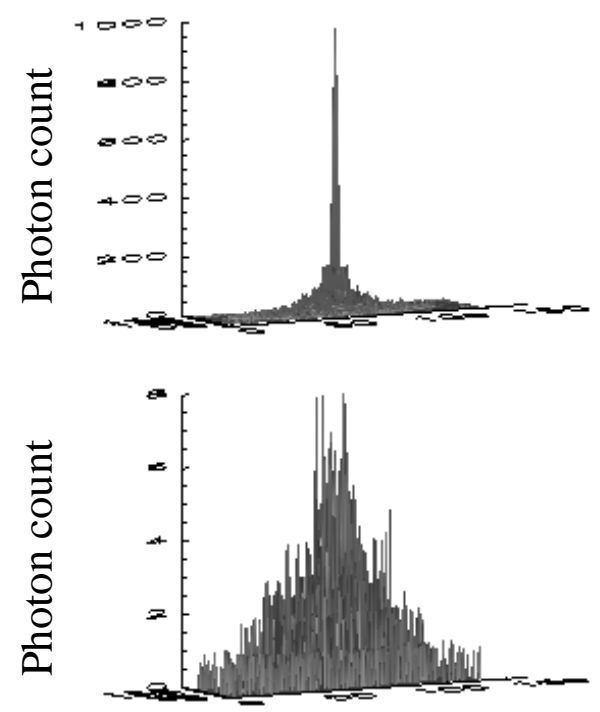

(d)

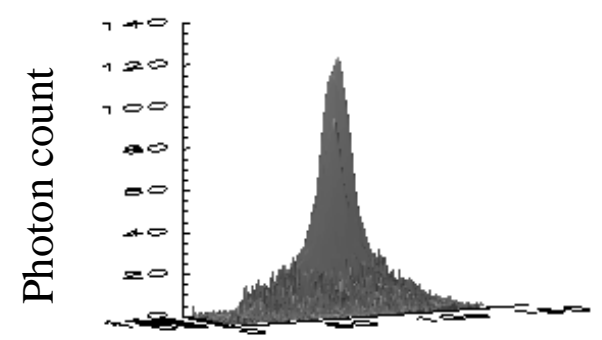

Fig. 7. Corrected phases (left) and images (right) obtained with an unresolved monochromatic source (frames a \& b) and with the object shown in Fig. 6, the galaxy M 51 (frames c \& d). Images are obtained after 1 iteration of the phasing algorithm for 500 a), 100,000, b), 5000 c) and 130000 d) photon events detected during the phasing sequence. The corresponding Strehl ratios are $0.48,0.97,0.38,0.96$. The residual slope of the phase pattern affects only the image position

\section{Conclusions}

Our simulations of co-phasing algorithms, using a triplet hierarchy, show convergence for both the cases of unresolved and resolved objects. With 3 different wavelengths, zero path difference has been achieved with initial errors of the order of $10 \mu \mathrm{m}$. Simulations with limited photon rates have shown that resolved images of bright stars are in principle obtainable with apertures of $10 \mathrm{~cm}$, using adaptive phasing at visible wavelengths. Arrays of large adaptive telescopes should extend the limiting magnitudes towards $m_{v}=20$ on Earth and $m_{v}=35$ in space. Possible refinements of these algorithms remain to be explored, but the simulations demonstrate the effectiveness of the triplet algorithm, used with image sharpness criteria, for phasing a multi-aperture array. This establishes the practical feasibility of obtaining high-resolution snapshot images on compact objects, with future interferometers spanning up to thousands of kilometers. In particular, it supports current projects towards obtaining resolved images of exoplanets with sufficient quality to see clouds, continents and vegetated areas (Boccaletti et al. 1999).

Acknowledgements. One of us (E.P.) would like to express his gratitude to Alastair Macgregor for his supervision in the early stages of this project. He would also like to thank Neal Jackson for his supervision and fruitful discussions during the continuation of this project at Jodrell Bank. 
We are very much indebted to Laurent Koechlin and Luc Arnold for their critical reading of the manuscript and useful suggestions. This work started at the University of Northumbria as a final year project and continued at the Observatoire of Haute Provence and NRAL, Jodrell Bank.

\section{References}

Angel J.R.P., Woolf N.J., 1997, ApJ 475, 373

Bacon R., et al., 1995, A\&A 113, 347

Baldwin J.E., et al., 1996, A\&A 306, L13-L16

Beckers J.M., 1997, Field of view considerations for telescope arrays. In Advanced technology optical telescopes III, 628 of 1986SPIE..628..255B, p. 255-260

Beckers J.M., 1997, Techniques for high angular resolution. In Instrumentation for large telescopes, Cambridge Contemporary Astrophysics. Cambridge University Press, Cambridge, CB2 1RP, p. 1-33

Boccaletti A., Riaud P., Moutou C., Labeyrie A., 1999, Snapshot coronagraphic imaging with a multi-aperture interferometer in space. Icarus (submitted)

Dyson F.J., 1978, Image processing and live optics. In Conference on Optical Telescopes of the Future, Geneva, Switzerland, December 12-15, 1977, A79 of 1978otf..conf..439D, p. 439-444

Hamaker J.P., O'Sullivan J.D., Noordam J.E., 1977, J. Opt.
Soc. Am. A 67(8), 1122-1123

Jennison R.C., 1958, MNRAS 118, 276-284

Labeyrie A., 1983, Construction of coherent arrays. In Workshop on ESO's Very Large Telescope, Cargese, Corse, France, May 16-19, 1983, 1983vlt..work..141D, p. 141-154 Labeyrie A., 1996, A\&A 118, 517-524

Labeyrie A., 1998, Direct searches: imaging, dark speckle and coronography. NATO ASI'98 In Planets outside the Solar System, Cargese, Corse, France, May 5-15

Muller R.A., Buffington A., 1974, J. Opt. Soc. Am. A 64(9), 1200-1210

Morel S., Koechlin L., 1998, Fringe tracking and a priori information on the optical path difference drift. In Astronomical Interferometry, 3350 of 1998SPIE.3350.1057M, p. 10571064

Roddier F., Roddier C., 1991, Appl. Opt. 30(11), 1325-1327

Ribak E., 1990, Telescope phasing by simulated annealing. In Amplitude and Intensity Spatial Interferometry, volume 1237 of 1990SPIE.1237..574R, p. 574-584

Shack R.B., Platt B.C., 1971, J. Opt. Soc. Am. A 61, 656

Traub W.A., 1986, Appl. Opt. 25, 528-532

Woolf N.J., 1997, Bringing planet finder closer: 1au missions for terrestrial planet finder, precursor and follow-on. In American Astronomical Society Meeting, 191, 64.04, 191 of 1997AAS...191.6404W, p. 6404W 